\title{
Análise de componentes principais para características de crescimento em bovinos de corte
}

\section{Principal components analysis for growth traits in beef cattle}

\author{
Carolina Amália de Souza Dantas Muniz ${ }^{1 *}$; Sandra Aidar de Queiroz²; \\ Arthur dos Santos Mascioli ${ }^{3}$; Lenira El Faro Zadra ${ }^{4}$
}

\section{Resumo}

Com o objetivo de estudar as inter-relações entre pesos e entre ganhos médios diários, utilizando-se a técnica de componentes principais, foram utilizadas 1663 observações de pesos ao nascimento (PN), a desmama ajustado para 230 dias (PD), ao ano ajustado para 365 dias (P365), ao sobreano ajustado para 550 dias de idade (P550) e ganho médio diário do nascimento à desmama (GND), da desmama a um ano de idade (G365) e dos 365 dias aos 550 dias de idade (G550) de um rebanho de animais cruzados F1, e um segundo arquivo contendo 320 observações das mesmas características de animais puros da raça Nelore. O modelo matemático para todas as características incluíram os efeitos de grupo de contemporâneos e os efeitos linear e quadrático da idade da vaca ao parto, enquanto o efeito fixo de raça foi considerado apenas para o arquivo de animais F1. Para os pesos, o primeiro componente principal contrastou animais mais pesados com animais mais leves após o nascimento, e explicou 79,0\% e 78,0\% da variação total para os arquivos contendo animais F1 e puros Nelore, respectivamente. O segundo componente principal comparou animais que atingiram maiores pesos na desmama com animais que foram mais pesados aos 550 dias de idade, explicando $13,5 \%$ e $15,5 \%$ da variação total para os arquivos de animais $\mathrm{F} 1$ e puros Nelore, respectivamente. A maior fonte de variação entre animais foi devida às diferenças em peso, seguida por diferenças nas idades nas quais os animais atingiram maiores pesos. Para o ganho médio diário nas diferentes idades, a variação entre animais foi principalmente devido às diferentes épocas de nascimento. O primeiro componente principal, explicou $52,0 \%$ e $56 \%$ da variação total para animais F1 e Nelore, respectivamente. Esse componente contrastou animais de maiores G365 com animais de maiores GND e G550, respectivamente, para animais F1 e Nelore.

Palavras-chave: Bovinos cruzados, ganho médio diário, peso ao nascer, peso à desmama, peso ao ano, peso ao sobreano

\footnotetext{
Abstract

The objetive of this research was to study the relation among body weight and average daily gain in different ages, using principal components analysis. Data on 1663 birth weight (BW), weaning weight adjusted to 230 days (WW), yearling weight adjusted to 365 days (YW), long yearling weight adjusted to 550 days (LYW), average daily gain from birth to weaning (AGW), average daily gain from weaning to 365 days (AGY) and average daily gain from 365 days weight to 550 day weight (AGL) from crossbred animals, and data on 320 observations of the same traits from straightbreed Nellore animals were analysed. The model included the fixed effects of breed (only crossbred data), contemporary

${ }^{1}$ Prof $^{\text {a }}$ do Dept ${ }^{\circ}$ de Zootecnia, Universidade Estadual de Londrina, UEL, Londrina, PR. E-mail: muniz@uel.br

${ }^{2}$ Prof $^{\mathrm{a}}$ do Dept $^{\mathrm{o}}$ de Zootecnia, Universidade Estadual Paulista Júlio de Mesquita Filho, Faculdade de Ciências Agrárias e Veterinárias, UNESP, Jaboticabal, SP. E-mail: saquei@unesp.br

${ }^{3}$ Prof. do Colegiado de Zootecnia, Universidade Federal de Goiás, UFG, Campus Jataí, Jataí, GO. E-mail: amascioli7@hotmail.com

${ }^{4}$ Pesquisadora, APTA, Estação Experimental de Zootecnia, Ribeirão Preto, SP. E-mail: lenira@apta.sp.gov.br
}

* Autor para correspondência 
group, and linear and quadratic effects of age at calving. For body weight in different ages, the first principal component contrasted heavier and light animals after birth and explained about $79,0 \%$ and $78,0 \%$ of the variation for data on crossbred and Nellore animals, respectively. The second principal component compared heavier animals at weaning and yearling weight those at long yearling weight . It explained around $13,5 \%$ and $15,5 \%$ of the total variation, respectively, for data on F1 and Nellore breed. The major source of variation among animals on the two data set for body weight was due to differences in weight followed by differences in the ages they got those weight. For the traits expressed as average daily gain, the variation among animals was due to differences in birth season, the first principal component explaining about $52,0 \%$ of the variation on crossbred animals. This component compared animal with higher AGY with those with higher AGW and AGL. For data on Nellore breed, the first component explain about $56,0 \%$ of the total variation and also compared animals with higher AGY with those with higher AGW and AGL.

Key words: Average daily gain, birth weight, crossbred animals, long yearling weight, yearling weight, weaning weight

\section{Introdução}

A identificação e seleção dos melhores genótipos são realizadas pela análise do desempenho de várias características de importância econômica. Entretanto, nos programas de melhoramento genético de bovinos de corte, faz-se necessário conhecer a importância e contribuição de cada uma destas variáveis, uma vez que a escolha dos critérios de seleção é imprescindível para o sucesso da atividade. Dessa forma, as técnicas de análise multivariada, em especial a de componentes principais, têm sido utilizadas para investigar a estrutura de covariâncias entre várias características estudadas, bem como as relações de dependência entre elas, o que auxilia muito o trabalho do profissional melhorista.

Muitas vezes a seleção ocorre em razão de uma determinada característica, entretanto, os componentes desta, bem como a correlação entre eles podem ser negligenciados. Além disso, a decisão das características a serem selecionadas pode ser influenciada por caracteres não relacionados aos objetivos que podem gerar respostas correlacionadas indesejáveis ao processo seletivo (CARVALHEIRO; CAVALCANTI, 2008).

De acordo com Morrison (1976), a análise de componentes principais consiste em representar a variabilidade resultante de $p$ variáveis aleatórias interdependentes pela de $q$ variáveis, sendo preferivelmente $q<p$ e combinações lineares das $p$ variáveis, tais que, após a transformação, sejam não correlacionadas entre si. De fato o que se busca são combinações lineares das variáveis originais (p) de modo que as novas $(q)$, os componentes principais, tenham variância máxima e sejam ortogonais entre si. Portanto, cada componente principal é uma combinação linear de todas as variáveis originais, independentes entre si e estimados com o propósito de reter, em ordem de estimação, o máximo de informação, em termos da variação total contida nos dados. Assim, a importância relativa de um componente é avaliada pela percentagem da variância total que ele explica. As variâncias dos componentes principais são os autovalores e os coeficientes das equações lineares de cada componente principal que transforma os dados originais são denominados autovetores. O primeiro autovalor a ser determinado corresponderá à maior porcentagem da variabilidade total presente e assim sucessivamente. Já, os autovetores são os resultados da participação das variáveis originais em cada componente Também, de acordo com Meyer (2006), os componentes principais de um grupo de $k$ efeitos correlacionados, são um grupo de $k$ variáveis com as funções lineares dos efeitos não correlacionados e que explicam sucessivamente o máximo da variação entre os $k$ efeitos.

Portanto, objetivou-se verificar a importância relativa de pesos e ganhos de peso de bovinos 
cruzados e puros da raça Nelore em diferentes idades, sobre a variabilidade dos indivíduos, utilizando-se a técnica de componentes principais.

\section{Material e Métodos}

Foram avaliados os pesos ao nascimento $(\mathrm{PN})$, à desmama, (padronizados para 230 dias de idade- PD), a um ano de idade (P365), aos 18 meses de idade (P550), e ganhos médios diários do nascimento à desmama (GND), da desmama a um ano de idade (G365) e dos 365 dias aos 550 dias de idade (G550). Os dados investigados neste estudo foram provenientes de 320 animais da raça Nelore e de 1663 animais cruzados F1, filhos de vacas Nelore. Os grupos genéticos cruzados (F1) foram constituídos por animais filhos de touros das raças Aberdeen Angus (AAN, 238 animais), Brangus (BN, 515 animais), Canchim (CN, 364 animais), Gelbvieh (GN, 244 animais) e Simental (SN, 302 animais). Os acasalamentos dos grupos genéticos das raças Aberdeen Angus e Gelbvieh foram apenas oriundos de inseminação artificial, enquanto os demais foram também de monta natural. Os animais pertencem à Fazenda Ivaé, situada no município de Amambai, Mato Grosso do Sul, localizada a 2310' de latitude sul e $55^{\circ} 15^{\prime}$ de longitude oeste, onde há duas estações climáticas bem distintas: a das águas (de outubro a março), e a seca(de abril a setembro). Os animais nasceram entre os anos de 1991 e 1994 e foram criados exclusivamente em regime de pasto, recebendo somente suplementação mineral. As pastagens eram formadas, na maior parte, por brachiárias (Brachiaria brizantha, B. decumbens e B. ruziziensis), com cerca de cinco anos de formação na ocasião da coleta dos dados e lotação média anual de 1,5 UA/ha.

As análises estatísticas, para os dois conjuntos de dados e para todas as características estudadas, incluíram os efeitos de grupo de contemporâneos (GC), compostos pelos animais de mesmo sexo, nascidos no mesmo ano e mês, e pelos efeitos linear e quadrático da idade da vaca ao parto, além do efeito fixo de raça, considerado apenas para o arquivo de animais F1.

As análises de componentes principais (CP) foram realizadas utilizando-se o procedimento PRINCOMP (SAS, 1999), onde foram considerados os resíduos e não os valores preditos.

\section{Resultados e Discussão}

As médias estimadas para as características PN, PD230, P365 e P550 para os animais cruzados foram respectivamente $30,24 \pm 2,75 \mathrm{~kg}, 192,47 \pm 18,15 \mathrm{~kg}$, $218,59 \pm 21,42,302,55 \pm 30,24 \mathrm{~kg}$.

Os coeficientes dos CP para as características de peso e de ganhos de peso dos animais cruzados são apresentados na Tabela 1. A maior parte da variância total $(92,5$ e 80,1\%) foi explicada pelos dois primeiros componentes dos pesos e ganhos de pesos, respectivamente, sendo ambos os componentes, bons índices para os programas de melhoramento genético em bovinos de corte. Para os pesos, o primeiro componente isolado explicou $79 \%$ de toda variação total, caracterizando sua importância destacada em relação aos outros e, portanto, representando um índice dos pesos dos bovinos cruzados. O fato de todos os coeficientes serem positivos indica que os animais foram contrastados dentro de uma mesma medida básica, ou seja, animais acima da média para algumas medidas e abaixo para outras, mostraram desvios positivos ou negativos, respectivamente (BROWN; BROWN; BUTTS, 1973).

Ao considerar que quanto maior o valor do componente em módulo maior seu poder discriminatório, ao interpretar a influência das características de peso sobre o referido componente, fica evidente que a importância dos pesos aumenta gradativamente com a idade do animal, demonstrando que os pesos de maior importância são aqueles mais próximos ao abate. Neste contexto, entendendo que os pesos obtidos nas idades inferiores compõem os pesos adjacentes superiores, 
este primeiro componente pode servir como um bom índice de critério de seleção, demonstrando que não há necessidade, por exemplo, de buscarem-se animais mais pesados ao nascimento, pois a variação de PN não é significativa. Entretanto, apesar da menor contribuição relativa dos pesos ao desmame e ao ano (43 e 55\%, respectivamente), no primeiro componente, eles demonstram-se imprescindíveis.

Tabela 1. Componentes principais das características pesos ao nascimento (PN), à desmama (PD), ao ano (P365) e ao sobreano (P550), e dos ganhos de pesos do nascimento à desmama (GND), da desmama aos 365 dias (G365) e dos 365 dias aos 550 dias de idade (G550) de bovinos cruzados.

\begin{tabular}{ccccc}
\hline & \multicolumn{3}{c}{ Componentes Principais } \\
\cline { 2 - 5 } Característica & CP1 & CP2 & CP3 & CP4 \\
\hline PN & 0,00 & $-0,00$ & $-0,00$ & 0,99 \\
PD & 0,43 & 0,82 & $-0,38$ & $-0,00$ \\
P365 & 0,55 & 0,09 & 0,83 & 0,00 \\
P550 & 0,71 & $-0,56$ & $-0,41$ & $-0,00$ \\
\hline \% Var. Total & 79,0 & 13,5 & 7,0 & 0,0 \\
\hline GND & $-0,27$ & $-0,03$ & 0,95 & \\
G365 & 0,86 & 0,42 & 0,26 & \\
G550 & $-0,40$ & 0,90 & $-0,09$ & \\
\hline \%Var. Total & & &
\end{tabular}

${ }^{1}$ Porcentagem da variância total.

Fonte: Elaboração dos autores.

O segundo componente principal, representando apenas $13,5 \%$ da variação total, possui importância acentuadamente menor, mas faz um contraste entre PD e P550, de modo que terá valor alto quando PD for elevado e P550 for baixo. Por outro lado, se o PD é baixo e o P550 é alto, este componente terá valor baixo, representando uma diferença dos pesos mencionados. Os baixos coeficientes de PN e P365 indicam que os valores destas variáveis não afetam o segundo componente, o qual pode estar evidenciando um contraste entre animais com crescimento diferenciado, ou seja, que atingiram maiores pesos ainda jovens, principalmente a desmama (PD), com aqueles de maior peso em idades mais avançadas, ao sobreano (P550). Além disso, evidencia-se a maior importância do PD $(82 \%)$, neste caso, caracterizando o contraste entre a maior ou menor precocidade de crescimento, ou então as taxas de maturação precoce em comparação com a tardia.
Já, o terceiro componente, com apenas 7\% da variação total, ortogonal aos anteriores, contrasta o bom desempenho de animais aos 12 meses com aqueles de maiores pesos nas idades adjacentes. $\mathrm{Ou}$ seja, o componente 3 destaca a maior variabilidade e contribuição do P365 que PD e P550, individualmente, e só será alto se o PD e o P550 forem baixos e P365 for alto. Uma vez que também foram utilizados os resíduos, ao invés dos valores preditos, é provável que parte das diferenças genéticas entre os animais tenha sido removida e assim, provavelmente animais mais pesados ao sobreano apresentaram crescimento compensatório, de modo que esta diferença não poderia ser atribuída ao potencial genético dos indivíduos, mas sim aos efeitos ambientais. Ao analisar a representatividade praticamente nula do quarto componente principal, que avalia apenas a variação de $\mathrm{PN}$, reafirma-se que não há necessidade de procurar animais com PN elevados, mas sim que os mesmos sejam sadios e 
tenham parâmetros na média de cada raça ou grupo genético.

Barbosa, Ledic e Deragon (1997) e Mascioli, Alencar e Fries (2000), que trabalharam com características de crescimento e morfológicas, em touros de várias raças zebuínas e bovinos da raça Canchim, respectivamente, observaram que a variação nas características estudadas foram bem sumarizadas por três componentes principais. O comportamento e os coeficientes dos componentes principais para as características de pesos nos estudos de Mascioli, Alencar e Fries (2000) foram muito semelhantes aos do presente estudo. Para estes autores, o comportamento e os coeficientes dos componentes principais das características de crescimento (pesos e ganhos de pesos) até dois anos de idade, foram muito semelhantes, de modo que os três primeiros explicaram aproximadamente
95\% da variância total, sendo que o primeiro (74\%), contrastando também, os animais que obtiveram maiores pesos após o nascimento. De maneira semelhante, o segundo (14\%) e o terceiro componente contrastaram animais que atingiram maiores pesos em idades mais jovens com aqueles que atingiram maiores pesos em idades posteriores. Barbosa, Ledic e Deragon (1997) verificaram que o processo seletivo favoreceu animais esguios, já Mascioli, Alencar e Fries (2000) verificaram que os pesos tornam-se mais importantes na medida que o animal envelhece.

O peso ao nascimento não foi relevante para os três primeiros componentes principais (Tabelas 1 e 2), caracterizando-se como uma característica que não apresenta grandes variações dentro dos rebanhos avaliados, além de não interferir no desempenho posterior ao nascimento.

Tabela 2. Componentes Principais das características pesos ao nascimento (PN), à desmama (PD), ao ano (P365) e ao sobreano (P550) e dos ganhos de pesos do nascimento à desmama (GND), da desmama aos 365 dias (G365) e dos 365 dias aos 550 dias de idade (G550) de bovinos da raça Nelore.

\begin{tabular}{ccccc}
\hline & \multicolumn{4}{l}{ Componentes Principais } \\
\cline { 2 - 5 } Característica & CP1 & CP2 & CP3 & CP4 \\
\hline PN & 0,00 & $-0,00$ & $-0,00$ & 0,99 \\
PD & 0,41 & 0,44 & 0,80 & 0,00 \\
P365 & 0,54 & 0,58 & $-0,60$ & $-0,00$ \\
P550 & 0,73 & $-0,68$ & 0,00 & $-0,00$ \\
\hline \%Var. Total ${ }^{1}$ & 78,0 & 15,5 & 6,0 \\
\hline Característica & CP1 & Componentes Principais & \\
\hline GND & 0,05 & $-0,11$ & CP3 & \\
G365 & $-0,49$ & 0,85 & 0,99 & \\
G550 & 0,87 & 0,50 & $-0,13$ & \\
\hline \%VT & 56,0 & 25,0 & 17,01 & \\
\hline
\end{tabular}

${ }^{1}$ Porcentagem da variância total.

Fonte: Elaboração dos autores.

Segundo Roso e Fries (1995), a determinação dos componentes da produção pode ter um significado econômico apreciável, uma vez que ao estudarem a influência de algumas características sobre a variabilidade dos bovinos Hereford, nas fases de desmama e sobreano os autores verificaram que a maior causa de variação foi devida a diferenças em tamanhos ou volumes corporais, seguida por 
diferenças referentes aos tipos de animais. De acordo com os coeficientes dos componentes principais nesse estudo, os resultados mostraram que no processo seletivo, o favorecimento de animais esguios pode ocorrer à custa de menor peso e massa muscular.

As relações entre puberdade, crescimento e tamanho foram estudadas por Baker et al. (1998), em bovinos cruzados com raças Angus, Brahman, Hereford, Holandês e Jersey, os autores verificaram que a maior parte da variação entre as raças foi devida à altura e não às características de desempenho. Entretanto, estes autores discutiram a maior suscetibilidade à influência ambiental para características de peso do que para medidas de tamanho.

Ao avaliar os diferentes objetivos das distintas fases de cria, recria e terminação da bovinocultura de corte, que é predominantemente realizada em sistemas de pastejo, pode-se inferir que para os sistemas de cria é interessante ter animais com maiores PD, entretanto, para os sistemas de recria e terminação, os animais de maior desempenho após a desmama devem ser priorizados.

Em relação às três características de ganho de peso dos bovinos cruzados (Tabela 1), os dois componentes principais explicaram $80 \%$ da variação total, sendo que o primeiro componente foi responsável por $52,9 \%$ da variação total. Este primeiro componente contrasta o G365 com os GND e G550 e, possivelmente está contrastando animais com ganhos médios diferentes em diferentes épocas do ano, ou seja, descrimina os animais que se apresentaram como bons ganhadores de peso nas épocas favoráveis versus os bons ganhadores em épocas desfavoráveis. Neste caso, o efeito aditivo seria o fator determinante da variabilidade entre indivíduos, o que poderia implicar na utilização preferencial de determinados touros conforme a época de nascimento de seus filhos. Além disso, os coeficientes deste componente demonstram que o G365 tem a maior contribuição para a variação total entre os animais, podendo estar associado a vários fatores, como a época de nascimento dos animais, habilidade materna da mãe, estresse do final da desmama, ou então a disponibilidade e qualidade das pastagens no período subsequente à desmama.

Possivelmente, os bezerros desmamados em pastagens com quantidade e qualidade baixas, sofrem maior estresse em relação aos desmamados em melhores condições de pastagem (MUNIZ; QUEIROZ, 1999). Mascioli, Alencar e Fries (2000) e Cardoso et al. (2003), estudando características de crescimento, também verificaram contrastes entre ganhos médios diários nas diferentes fases de crescimento, argumentando que os resultados poderiam estar contrastando épocas favoráveis (no período ano-sobreano) e desfavoráveis (sobreanodois anos) e a capacidade do animal em ganhar peso nestas épocas.

O segundo e o terceiro componentes para os ganhos de peso de bovinos cruzados explicaram 28,1 e 19\% da variação total (Tabela 1), respectivamente, e funcionam como um índice de importância dos três períodos de ganho, sendo que o segundo componente não considera a contribuição do GND e o terceiro não considera o G550. O segundo CP é um índice que destaca os bons ganhadores de peso nos períodos pós-desmama (G365 e G550), valoriza o G365 e, principalmente o G550, período de maior importância em relação à variação dos dados. Já, o terceiro CP considera que $19 \%$ da variação deve-se à contribuição do GND e em menor peso do G365, destacando assim, os efeitos da habilidade materna.

Esses resultados, especialmente dos primeiros componentes principais, vão de encontro à tendência atual dos programas de melhoramento genético, que valorizam a precocidade dos animais que alcançam os índices em menor tempo, em relação ao desempenho produtivo de animais ainda jovens, esperando assim reflexos positivos e preponderantes em relação à precocidade reprodutiva.

O comportamento e a magnitude dos valores dos componentes principais para os pesos e ganhos 
de peso de bovinos da raça Nelore foram similares aos dos bovinos cruzados, sendo que 78\%, 15,5\% e $6 \%$ da variação total dos dados foram explicadas pelos três primeiros componentes, respectivamente (Tabela 2). O primeiro CP para peso desconsidera o $\mathrm{PN}$, mas valoriza em ordem crescente o PD, P365 e o P550, ressaltando que o índice busca animais mais pesados à medida que a idade aumenta.

A importância em relação à variação total e comportamento do segundo CP foi semelhante ao relatado para os animais cruzados (Tabela 1), entretanto, a magnitude dos coeficientes foi diferente, e contrastou o PD e P365 com o P550. Dessa maneira, este componente valoriza os animais com bom desempenho até um ano de idade, ou então, aqueles que não se desenvolveram bem até essa idade, mas que possuem um bom desempenho no período posterior, provavelmente caracterizado por um ganho compensatório.

Diferentemente dos animais cruzados, o P365 nos animais Nelore tem uma boa contribuição para o segundo $\mathrm{CP}$, evidenciando que uma pequena parcela da variação total $(15,5 \%)$ é explicada pelo bom desempenho até um ano de idade. Além disso, este componente pode também estar valorizando o contraste entre animais com crescimento diferenciado, ou seja, animais que atingiram maior peso em idades mais jovens, principalmente aos 12 meses de idade, com aqueles de maior peso em idades mais avançadas, ao sobreano (P550). Este CP permitiu visualizar a maior contribuição de $\mathrm{PD}$ para os animais cruzados (Tabela 1), possivelmente em função da melhor habilidade materna (produção de leite) das fêmeas cruzadas em relação aos animais Nelore (Tabela 2). Além disso, percebe-se uma variância maior para P365 em animais Nelores do que em cruzados.

Com relação ao terceiro $\mathrm{CP}$ dos animais Nelore, evidencia-se que apenas $6 \%$ de toda variação é atribuída ao contraste entre PD e P365, desconsiderando o P550. Comparado ao mesmo CP dos animais cruzados, observou-se que os sinais foram contrários nesse grupamento. Assim, este $\mathrm{CP}$ comparou e animais que atingiram maiores $\mathrm{PD}$ com os de menores P365, sugerindo que os animais que atingiram os maiores PD não foram aqueles que tiveram os melhores desempenhos de P365 e vice-versa. Assim, pode-se inferir que pequena parte da variação total dos pesos, em bovinos puros Nelore, é explicada pelo bom desempenho de PD e que a mesma porcentagem de variação em bovinos cruzados é explicada pelo maior desempenho do P365, caracterizando que existem diferenças de desempenho, até a idade de sobreano, entre os cruzados e Nelores.

Ao comparar as características de ganho de peso em Nelore, nota-se que as porcentagens das variâncias totais explicadas pelos três componentes foram semelhantes aos obtidos para os animais cruzados, entretanto o comportamento e as magnitudes dos coeficientes de todos os componentes diferiram. $\mathrm{O}$ primeiro CP desconsiderou a influência do GND, mas contrastou o G365 e G550 de animais da raça Nelore, valorizando os animais com maiores G550 versus aqueles de maiores G365, caracterizando que a maior parte da variação total (56\%) é oriunda deste CP.

De maneira análoga aos animais cruzados, o segundo e o terceiro componentes para os ganhos de peso em bovinos Nelore explicaram 25 e $17 \%$ da variação total, respectivamente. Porém, para o segundo CP nota-se uma contribuição maior do G365 seguida pela do G550, em contraste com uma pequena contribuição do GND. Estas importâncias foram contrárias para o mesmo componente nos animais cruzados (Tabela 1), em que se enfatizou mais o G550 do que o G365, desconsiderando o GND. Deste modo, este CP é um índice que destaca os bons ganhadores de peso nos períodos pós-desmama (G365 e G550), valorizando, principalmente, o G365, período de maior importância em relação à variação dos dados. Já, o terceiro CP considere que $17 \%$ da variação são principalmente devido à contribuição 
do GND e em menor peso do G365, destacando assim, os efeitos da habilidade materna.

Neste contexto, ao avaliar os sinais e as ênfases dos componentes dos ganhos de peso em animais puros Nelore em comparação aos cruzados, percebese maior variabilidade e contribuição do GND para os cruzados do que para os puros. Adicionalmente, os animais puros Nelore provavelmente sentem mais acentuadamente os efeitos da desmama do que os cruzados, uma vez que os ganhos na fase pós-desmama (G365) contribuíram negativamente $(-0,49)$ no primeiro $\mathrm{CP}$, quando comparado ao mesmo CP dos animais cruzados. Entretanto a contribuição do G550 para explicar a variação total em animais Nelore foi maior e positiva $(0,87)$, que para os cruzados $(-0,40)$, indica que os animais cruzados apresentam maiores variações de pesos nas idades mais avançadas, diferentemente dos Nelore, que são mais adaptados e que possivelmente, sentem menos os efeitos ambientais.

\section{Conclusões}

A maior causa da variação entre animais, tanto puros como cruzados, foi devida às diferenças em pesos após o nascimento.

O desenvolvimento dos animais foi diferenciado nas fases de crescimento, principalmente com relação às características PD, P365 e P550.

A principal causa de variação entre animais, quando se considera o ganho de peso, foi devida às diferentes épocas de nascimento, evidenciando a importância do estabelecimento da estação de monta.

\section{References}

BAKER, J. F.; STEWART, T. S.; LONG, C. R.; CARTWRIGHT, T .C. Multiple regression and principal components analysis of puberty and growth in cattle. Journal of Animal Science, Chanpaingn, v. 66, n. 9, p. 2147-2158, sept. 1988.
BARBOSA P. F.; LEDIC, I. L.; DERAGON, L. A. G. Análises de componentes e fatores principais de características morfológicas, peso, idade e circunferência escrotal em touros zebu. In: REUNIÃO ANUAL DA SOCIEDADE BRASILEIRA DE ZOOTECNIA, 34., 1997. Juiz de Fora. Anais... Juiz de Fora: SBZ, 1997. p. 235-237.

BROWN, J. E.; BROWN, C. J.; BUTTS, W. T. Evaluation relationships among immature measures of size, shape, and performance of beef bulls. I Principal components as measures of size and shape in young Hereford and Angus bulls. Journal of Animal Science, Champaingn, v. 36, n. 6, p. 1010-1020, jun. 1973.

CARDOSO, V.; ROSO, V. M.; SEVERO, J. L. P.; QUEIROZ, S. A; FRIES, L. A. Formando lotes uniformes de reprodutores múltiplos e usando-os em acasalamentos dirigidos em populações Nelore. Revista Brasileira de Zootecnia, Viçosa, MG, v. 32, n. 6, p. 834-842, 2003.

CARVALHEIRO, R.; CAVALCANTI, J. R. O biótipo dos animais do seu rebanho está de acordo com o seu objetivo de seleção? In: SIMPÓSIO BRASILEIRO DE MELHORAMENTO ANIMAL, 7., 2008, São Carlos. Anais... São Carlos: Sociedade Brasileira de Melhoramento Animal, 2008. CD-ROM.

MASCIOLI, A. S.; ALENCAR, M. M.; FRIES, L. A. Estimativas de parâmetros genéticos e fenotípicos e análise de componentes principais para características de crescimento na raça Canchim. Revista Brasileira de Zootecnia, Viçosa, MG, v. 29, n. 6, p. 1654-1660, 2000.

MEYER, K. To have your steak and eat it: genetic principal component analysis for beef cattle data. In: WORLD CONGRESS ON GENETICS APPLIED TO LIVESTOCK PRODUCTION, 8., 2006, Belo Horizonte. Anais... Belo Horizonte: SBMA, 2006. p. 13-18. CDROM.

MORRISON, D. F. Multivariate statistical methods. 2. ed. New York: McGraw-Hill, 1976. 416 p.

MUNIZ, C. A. S. D.; QUEIROZ, S. A. Avaliação de características de crescimento pós-desmama de animais Nelore e seus cruzados. Revista Brasileira de Zootecnia, Viçosa, MG, v. 28, n. 4, p. 713-720, 1999.

ROSO, V. M.; FRIES, L. A. Componentes principais em bovinos da raça Polled Hereford à desmama e sobreano. Revista Brasileira de Zootecnia, Viçosa, MG, v. 24, n. 5, p. 728-735, 1995.

STATISTICAL ANALYSIS SYSTEM - SAS. Statistical analysis system user's guide: stat, version 6.11. Carry: SAS Institute, 1999. 